\title{
Considering what we know, diagnostic yield and postoperative mortality associated with surgical lung biopsy for evaluation of interstitial lung diseases are good ... we think ...
}

\author{
Katie S. Nason, MD, MPH
}

See related article on pages 1394-401.

In their article, "Diagnostic Yield and Postoperative Mortality Associated with Surgical Lung Biopsy for Evaluation of Interstitial Lung Diseases: A Systematic Review and Meta-Analysis," Dr Qian and colleagues ${ }^{1}$ use a systematic literature review and meta-analysis to expand our understanding of the role for invasive procedures in this high-risk population. The authors are to be congratulated for tackling a difficult topic that is of great interest to thoracic surgeons, who must weigh the risks and benefits and accept the burden of potential mortality when discussing options with patients and their families. It is also a topic that is difficult to examine because of the wide range of underlying patient conditions present at the time of the biopsy (eg, intubated, immunocompromised, hospitalized vs outpatient) and the difficulty in accruing large numbers of patients into any one study. As such, it is well suited for examination by systematic review.

A total of 23 articles met criteria for inclusion. The diagnostic yield was $95 \%$ (range, $42 \%-100 \%$ ). In the 8 studies reporting influence on treatment algorithm, the proportion of cases leading to a change in treatment ranged from 42 to $90 \%$. The most common diagnosis after lung biopsy was interstitial pulmonary fibrosis (IPF) $(34 \%)$. Diagnostic accuracy was independent of the approach to surgical lung biopsy (ie, thoracoscopy vs thoracotomy). Pooled mortality at 90 days was $3.6 \%$, with significant heterogeneity across studies. This heterogeneity in mortality was likely due to differences in exclusion criteria between articles, including immunocompromised status, dependence on mechanical ventilation, and severe respiratory dysfunction, which were all associated with

From the University of Pittsburgh, Pittsburgh, Pa.

Dr Nason's work is supported by Award Number K07CA151613 from the National Cancer Institute. The content is solely the responsibility of the authors and does not necessarily represent the official views of the National Cancer Institute or the National Institutes of Health.

Disclosures: Author has nothing to disclose with regard to commercial support.

Received for publication Feb 20, 2015; accepted for publication Feb 20, 2015; available ahead of print March 24, 2015

Address for reprints: Katie S. Nason, MD, MPH, University of Pittsburgh, 5200 Centre Ave, Suite 715, Shadyside Medical Building, Pittsburgh, PA 15232

(E-mail: nasonks@upmc.edu).

J Thorac Cardiovasc Surg 2015;149:1402-3

$0022-5223 / \$ 36.00$

Copyright (c) 2015 by The American Association for Thoracic Surgery

http://dx.doi.org/10.1016/j.jtcvs.2015.02.035 higher mortality rates. Mortality was $8 \%$ with open lung biopsy compared with $2.9 \%$ with thoracoscopy, but was not significantly different in the 2 randomized trials included in the review. Deaths were more common in patients with IPF in 2 of 3 studies providing comparisons by diagnosis. On the basis of the analysis by Qian and colleagues, ${ }^{1}$ surgical lung biopsy was recommended for patients with signs and symptoms suggestive of, but not definitive for IPF, whereas caution was urged when considering surgical lung biopsy in patients with the significant risk factors for mortality listed earlier.

These recommendations are consistent with recently published guidelines for idiopathic pulmonary fibrosis ${ }^{2}$ and idiopathic interstitial pneumonia. ${ }^{3}$ Because thoracic surgeons are frequently asked to perform surgical biopsies in high-risk patients, a comprehensive understanding of the guidelines is critical. For example, current guidelines suggest that surgical biopsy is no longer essential for diagnosis in certain clinical settings, such as IPF. Combined with a thorough examination that includes review of occupational, environmental, family, medication, and comorbidity histories, and cardiopulmonary physiology testing, a high-resolution computed tomography (HRCT) scan showing usual interstitial pneumonia (UIP) is now considered sufficient for the diagnosis of IPF. ${ }^{2}$ In this setting, HRCT was associated with positive predictive values for UIP ranging from $90 \%$ to $100 \%$. However, in the absence of diagnostic imaging criteria for UIP, specifically honeycombing, surgical lung biopsy with interpretation by an expert pathologist is necessary and should be performed to further define patients with possible UIP. ${ }^{4}$ The guidelines emphasize interpretation of the data by radiologists, pulmonary physicians, and pathologists with expertise in the field in a multidisciplinary fashion, as well as the necessity to integrate clinical data, including treatment response, in assigning diagnosis., Comprehensive application of this approach will delineate circumstances in which a surgical biopsy will be more informative than HRCT and when a surgical biopsy is not necessary. ${ }^{3}$

The current systematic review ${ }^{1}$ highlights the limitations of the available literature on the diagnostic yield and safety of surgical lung biopsy. Despite well-described 
potential risk factors for interstitial lung disease, including cigarette smoking, environmental exposures, microbial agents, gastroesophageal reflux, and genetic factors, ${ }^{2}$ the data regarding these subsets in the published literature are limited. Likewise, factors influencing mortality were not reported or used as exclusion criteria to create a more homogeneous population. For future studies, it will be important to ensure that key variables, including preoperative pulmonary function tests, number of biopsies obtained, location of biopsies, time from onset of illness to biopsy, presence of active pneumonia or antibiotic use, immunosuppressed status, intubation status, approach to biopsy, and other relevant variables, are included whenever possible. In addition, if patient numbers allow, analysis of the key outcomes, including diagnostic yield, morbidity, and mortality, should be stratified by these variables to further elucidate factors influencing those outcomes.

Through standardization of reporting and knowledge of guidelines for surgical lung biopsy and variables influencing diagnostic yield of surgical biopsy and operative morbidity and mortality, thoracic surgeons will be properly armed to optimize patient outcome while minimizing harm. Given the small numbers of patients at any one institution, the development of a multiinstitutional, international prospective registry may be needed to provide the necessary data to fully understand the numerous variables affecting diagnostic yield and mortality after surgical lung biopsy for interstitial lung disease.

\section{References}

1. Qian H, Qun L, Jiaxing X, Lulu W, Liyue L, Xiaoxian Z, et al. Diagnostic yield and postoperative mortality associated with surgical lung biopsy for evaluation of interstitial lung diseases: a systematic review and meta-analysis. J Thorac Cardiovasc Surg. 2015;149:1394-401.e1.

2. Raghu G, Collard HR, Egan JJ, Martinez FJ, Behr J, Brown KK, et al. An official ATS/ERS/JRS/ALAT statement: idiopathic pulmonary fibrosis: evidence-based guidelines for diagnosis and management. Am J Respir Crit Care Med. 2011; 183:788-824.

3. Travis WD, Costabel U, Hansell DM, King TE Jr, Lynch DA, Nicholson AG et al. An official American Thoracic Society/European Respiratory Society statement: update of the international multidisciplinary classification of the idiopathic interstitial pneumonias. Am J Respir Crit Care Med. 2013;188: 733-48.

4. Wells AU. The revised ATS/ERS/JRS/ALAT diagnostic criteria for idiopathic pulmonary fibrosis (IPF)-practical implications. Respir Res. 2013;14(Suppl 1):S2. 